\title{
Efficacy and safety of $N$-acetylcysteine therapy for idiopathic pulmonary fibrosis: An updated systematic review and meta-analysis
}

\author{
FANCHAO FENG $^{1 *}$, JIARUI ZHANG ${ }^{2 *}$, ZHICHAO WANG $^{1}$, QI WU ${ }^{1}$ and XIANMEI ZHOU ${ }^{1,3}$ \\ ${ }^{1}$ Department of Respiratory Medicine, Affiliated Hospital of Nanjing University of Chinese Medicine, Nanjing, \\ Jiangsu 210029; ${ }^{2}$ State Key Laboratory Cultivation Base for TCM Quality and Efficacy, School of Medicine and \\ Life Science, Nanjing University of Chinese Medicine, Nanjing, Jiangsu 210023; ${ }^{3}$ Department of Respiratory Medicine, \\ Jiangsu Province Hospital of Chinese Medicine, Nanjing, Jiangsu 210029, P.R. China
}

Received November 26, 2018; Accepted April 12, 2019

DOI: $10.3892 /$ etm.2019.7579

\begin{abstract}
Idiopathic pulmonary fibrosis (IPF) is a progressive and ultimately fatal lung disease with poor prognosis and limited treatment options. $N$-acetylcysteine (NAC), an anti-oxidant drug, has promising potential in the treatment of IPF. In the present systematic review and meta-analysis, the efficacy and safety of NAC for IPF were investigated. The following databases were comprehensively searched for relevant studies published until August 2018: Pubmed, Embase, Cochrane library, Chinese National Knowledge Infrastructure, Wangfang Database, VIP and the Chinese Biology Medical Database. A total of 21 controlled trials assessing the efficacy and safety of NAC therapy for IPF were identified and primary outcomes [forced vital capacity (FVC), adverse side effects] and secondary outcomes [diffusing capacity for carbon monoxide (DLCO) and its percentage predicted value (DLCO\%), vital capacity (VC), partial arterial oxygen pressure $\left(\mathrm{PaO}_{2}\right), 6-\mathrm{min}$ walking distance test and mortality] were extracted for the meta-analysis. The risk ratio and mean difference or standardized mean difference with $95 \%$ confidence interval were calculated using RevMan 5.3 software. Analysis of the pooled data revealed that, compared with control treatments (routine treatment or drugs other than anti-oxidants), NAC therapy reduced the decline in lung function, as indicated by the FVC and DLCO, and slowed the progression of the disease, as indicated by the $\mathrm{PaO}_{2}$, while complications and mortality were similar. These results suggest good efficacy, tolerability
\end{abstract}

Correspondence to: Professor Xianmei Zhou, Department of Respiratory Medicine, Jiangsu Province Hospital of Chinese Medicine, 155 Hanzhong Road, Nanjing, Jiangsu 210029, P.R. China E-mail: zhouxianmeijs@aliyun.com

*Contributed equally

Key words: $N$-acetylcysteine, idiopathic pulmonary fibrosis, efficacy, safety, meta-analysis and safety of the treatment. Furthermore, subgroup analysis revealed that combined therapy including NAC for IPF might be more effective than NAC monotherapy, while oral administration of NAC was safer than inhalation. In conclusion, the results of the present review and meta-analysis provide important information that may serve as a guide regarding NAC therapy for IPF in clinical practice.

\section{Introduction}

Idiopathic pulmonary fibrosis (IPF) is a specific chronic, progressive and fibrosing interstitial lung disease $(1,2)$, where the normal lung parenchyma is progressively replaced by an altered extracellular matrix with destruction of the alveolar architecture. These changes lead to decreased lung compliance, impaired gas exchange and, ultimately, respiratory failure and death (3). IPF occurs worldwide and its incidence has been increasing over time, with $\sim 2.8-9.3$ cases per 100,000 people in Europe and North America in 2012 (4). The IPF-associated mortality rate is high and the median survival time after diagnosis is 2-4 years $(3,5)$. However, the pathogenesis of IPF has remained to be fully elucidated.

IPF is a consequence of multiple integrating genetic and environmental factors, which cause a cascade of injury of the alveolar epithelium (6,7). Most of these factors disrupt the oxidant/anti-oxidant balance in the lung $(8,9)$ and evidence has indicated that patients with IPF exhibit higher levels of oxidative biomarkers and lower levels of anti-oxidant biomarkers (10). $\mathrm{N}$-acetylcysteine (NAC), an alternative anti-oxidant, has been demonstrated to improve the glutathione homeostasis in the human body (11). Furthermore, it was reported to decrease inflammation and collagen deposition in a mouse model of bleomycin-induced lung fibrosis (12).

However, according to the clinical trials that have emerged in previous years, the efficacy of NAC therapy for IPF remains controversial. While certain studies have suggested that NAC therapy provides a benefit for patients with IPF and slows the deterioration of lung function, particularly the decline in forced vital capacity (FVC) (13-17), others have indicated no benefit of this treatment for IPF (18-20). In addition, the results 
of previous meta-analyses on the efficacy of NAC therapy for IPF are contradictory. Sun et al (21) determined that the only significant effects of NAC therapy were to decrease the percentage of predicted $\mathrm{VC}$ and improve the 6-min walking distance test (6MWT), whereas Kandhare et al (22) indicated that anti-oxidant therapy was only significantly associated with the percentage of predicted $\mathrm{VC}$ and changes in the percentage of predicted diffusing capacity for carbon monoxide (DLCO) in IPF patients.

Considering this conflicting evidence, an updated and comprehensive systematic review and meta-analysis of the efficacy and safety of pharmaceutical treatments for IPF including NAC were performed in order to provide information that may guide further clinical decisions regarding this therapy.

\section{Materials and methods}

Data resources and search strategies. A comprehensive search for relevant studies on NAC therapy for IPF published until August 2018 was performed in appropriate electronic databases and sources, including Pubmed, EMbase, the Cochrane library, the Chinese National Knowledge Infrastructure, the Wangfang Database, the VIP Database and the Chinese Biology Medical Database, by two independent reviewers (FF and JZ). The language was restricted to Chinese and English. The following keywords or free terms were used: ('acetylcysteine' or ' $N$-acetylcysteine' or 'NAC') and ('idiopathic pulmonary fibrosis' or 'IPF' or 'usual interstitial pneumonia'). All clinical studies except case reports were selected for analysis. The bibliographies of primary studies, as well as the references listed in the selected articles, were also searched for further relevant publications.

Inclusion and exclusion criteria. The inclusion criteria for studies were as follows: i) Controlled trials comparing a NAC-treated group with a control group that received routine treatment or drugs other than anti-oxidants, e.g., lecithinized superoxide dismutase, for the treatment of IPF. ii) The diagnosis of IPF was in accordance with an official statement of the American Thoracic Society/the European Respiratory Society/the Japanese Respiratory Society/the Latin American Thoracic Association Clinical Practice Guideline (2). iii) The following outcome measures were reported in the trials: FVC and adverse effects were regarded as primary outcomes, while secondary outcomes that were also retrieved included DLCO, the percentage predicted DLCO (DLCO\%), VC, partial arterial oxygen pressure $\left(\mathrm{PaO}_{2}\right), 6 \mathrm{MWT}$ and mortality.

Studies that did not meet the inclusion criteria mentioned above were excluded. In addition, review articles, animal experiments, duplicated publications, studies with inappropriate interventions and studies with insufficient useful data were not considered.

Assessment of methodological quality. The methodological quality of all of the included trials was independently assessed in duplicate by two reviewers (FF and JZ). This quality assessment was according to a scoring system from 0 to 14 , which evaluated the following aspects: Randomization, blinding, analysis, patient selection, comparability of groups at baseline, extent of follow-up, treatment protocol, co-interventions and outcomes (23). A consensus between the two reviewers was reached for individual category scores. This assessment strategy has been widely used in previous meta-analysis publications $(24,25)$.

Clinical outcomes. All data were extracted by two independent reviewers ( $\mathrm{FF}$ and $\mathrm{ZW}$ ) and the discrepancies were resolved by discussion with a third expert adjudicator (XZ). The primary outcomes were FVC and adverse effects, while DLCO, DLCO $\%, \mathrm{VC}, 6 \mathrm{MWT}, \mathrm{PaO}_{2}$ and mortality were the secondary outcomes.

Subgroup analysis. Pre-specified subgroup analyses were performed to explore the potential causes of heterogeneity in the effects of NAC therapy on the primary and secondary outcomes. It was hypothesized that the treatment efficacy and safety of NAC is greater in trials using i) NAC combined therapy vs. NAC monotherapy (due to the potential pharmacokinetic synergy achieved with combined therapy); ii) a higher NAC dose (1,800 mg per day) rather than below; iii) NAC aerosol administration (due to the direct anti-oxidant effects in alveoli); iv) those with a lower methodological quality (as studies with a higher methodological quality tend to exhibit more modest treatment effects).

Statistical analysis. All data were analyzed using RevMan 5.3 software (The Cochrane Collaboration). Mean differences (MDs) or standardized MDs (SMDs) with $95 \%$ confidence intervals (95\% CIs) were determined in the statistical analysis of continuous variables, whereas pooled risk ratio (RR) with 95\% CIs were used for dichotomous variables. Clinical and methodological heterogeneities among studies were analyzed using the $\chi^{2}$ and $\mathrm{I}^{2}$ tests. If the $\mathrm{I}^{2}$ value was $>50 \%$ and/or $\mathrm{P}<0.1$ for pooled studies, a random-effects model was used for the meta-analysis. Otherwise, a fixed-effects model was used. Outcomes were calculated using $\mathrm{P}$-values and $\mathrm{P}<0.05$ was considered to indicate statistical significance. As $>10$ studies were included in the present meta-analysis, funnel plots were drawn for the evaluation of potential publication bias with regard to outcomes. While publication bias may result in asymmetric funnel plots, clinical or methodological heterogeneity among studies affects the shape of the funnel plots. Therefore, publication bias was further evaluated by Egger's test using Stata 14.0 software (StataCorp.).

\section{Results}

Data collection. Initially, 961 potentially relevant articles were identified during the initial search of the seven databases. After removal of duplicated articles, the titles and abstracts of the remaining 734 articles were screened, following which 436 review articles, 92 animal or in vitro studies, eight case reports, three publications in German, 129 studies without relevant outcomes, five studies without a control group and 26 studies with inappropriate controls were excluded. Subsequently, the full-text of 35 articles was screened; this led to the exclusion of 14 articles due to repeated data, inappropriate controls or lack of assessable outcomes. Finally, a total of 21 eligible studies published between 2005 and 2016 were included in the present 

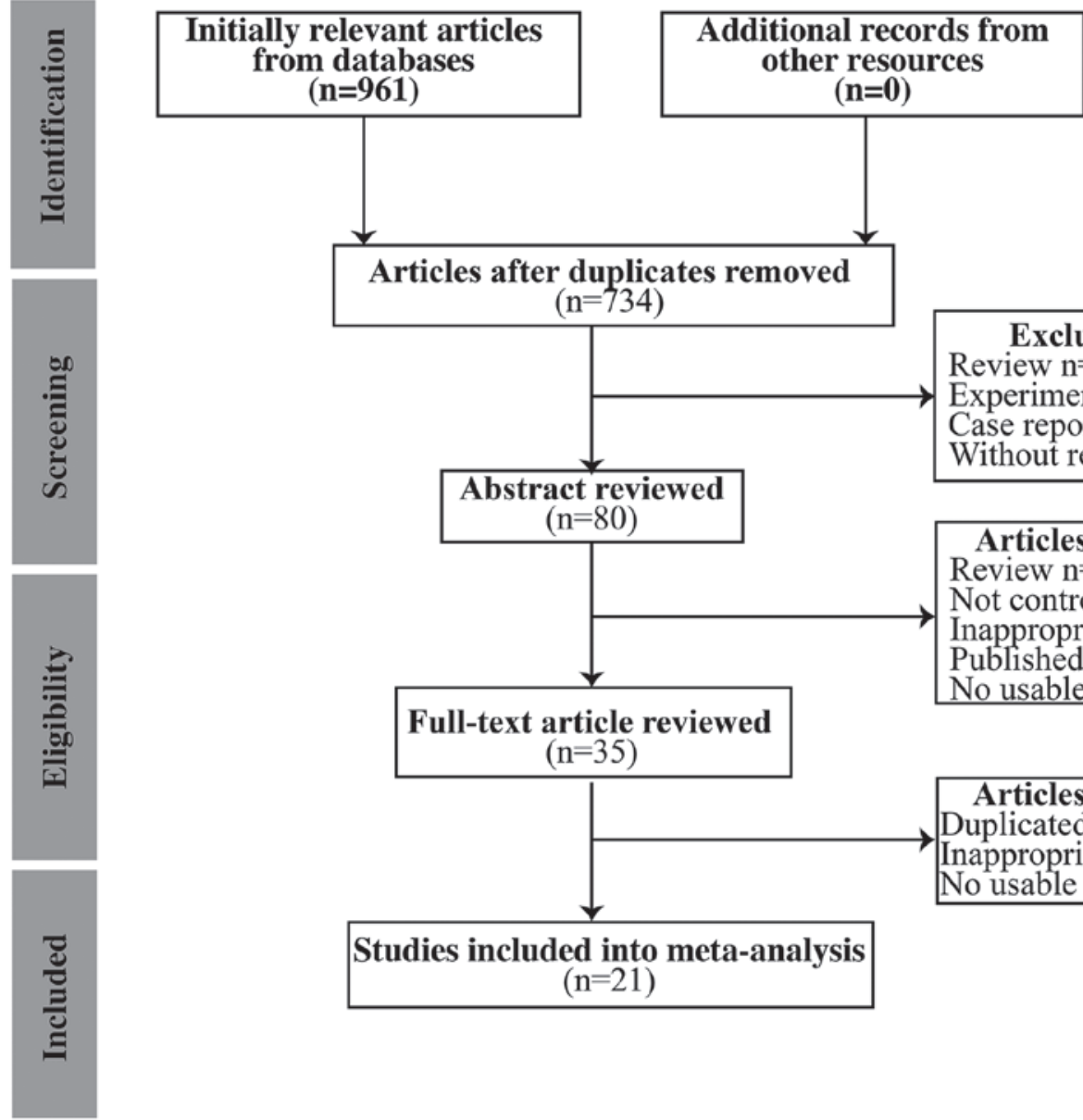

Excluded by title $n=654$

Review $\mathrm{n}=426$

Experimental study $n=92$

Case report $\mathrm{n}=8$

Without relevant outcomes $n=128$

Articles excluded $n=45$

Review $\mathrm{n}=10$

Not control study $\mathrm{n}=5$

Inappropriate control $\mathrm{n}=26$

Published in German $n=3$

No usable outcomes $n=1$

Figure 1. Flow chart illustrating the selection of studies for an updated systematic review and meta-analysis of the safety and efficacy of $N$-acetylcysteine therapy for idiopathic pulmonary fibrosis.

meta-analysis (13-20,26-38). A flow chart depicting the selection process is presented in Fig. 1.

Characteristics of the studies included. Among the 21 eligible studies, 13 were performed in China and eight in the United States, Japan or various European countries. Overall, 1,354 patients (including 695 patients who received NAC therapy and 659 who received other therapies) were identified. NAC was orally administered in all but five Japanese studies, where NAC was administered via inhalation. The most commonly used oral dose [76.19\% (16/21)] was $600 \mathrm{mg}$ three times a day $(1,800 \mathrm{mg}$ per day). Combined therapy frequently included corticosteroids (13 studies) and pirfenidone (two studies). The duration of treatment ranged from 3 to 15 months. The demographic and clinical baseline characteristics of the included studies, comprising the first author, year of publication, type of study, patient number and outcomes, are presented in Table I.

Assessment of methodological quality. Of the studies included, 18 were randomized controlled trials, two were case-control studies and one was a retrospective study. Only four of the 18 randomized controlled trials provided details about the randomization method. These studies were designed as randomized, double-blinded, placebo-controlled trials that declared the concealment of treatment allocation and blinding procedures. A total of 9 studies included the drop-out data and provided the detailed information. According to the quality scoring, the mean methodological quality score of an individual trial was 7.9, while the median score was 7 (range, 6-12). The individual scores for each trial are provided in Table I.

\section{Meta-analysis of primary outcomes}

Overall effect on FVC. The FVC was evaluated for a total of 470 patients in nine studies $(14,15,18,19,27,28,31,35,37)$. As presented in Fig. 2A, these studies had moderate heterogeneity $\left(\mathrm{I}^{2}=55 \%, \mathrm{P}=0.02\right)$; therefore, a random-effects model was used to analyze the data. Meta-analysis of the studies revealed that NAC treatment reduced the decline in FVC (MD, 0.26; 95\% CI, 0.10-0.41; P=0.001).

Overall adverse effects. A total of 12 studies $(13,14$, 16,18-20,27,31,33,36-38), including 1,003 patients, reported on the adverse effects of NAC therapy. The heterogeneity among studies $\left(\mathrm{I}^{2}=41 \%, \mathrm{P}=0.07\right)$ warranted the use of a random-effects model. The meta-analysis did not reveal any significant difference (RR, 1.08; 95\% CI, 0.84-1.38; $\mathrm{P}=0.57$ ) in adverse effects between the NAC therapy and control groups (Fig. 2B).

Meta-analysis of secondary outcomes

Overall effect on DLCO. A pooled analysis of DLCO data from seven studies $(13,27,28,31,34,36,37)$ was performed. A 


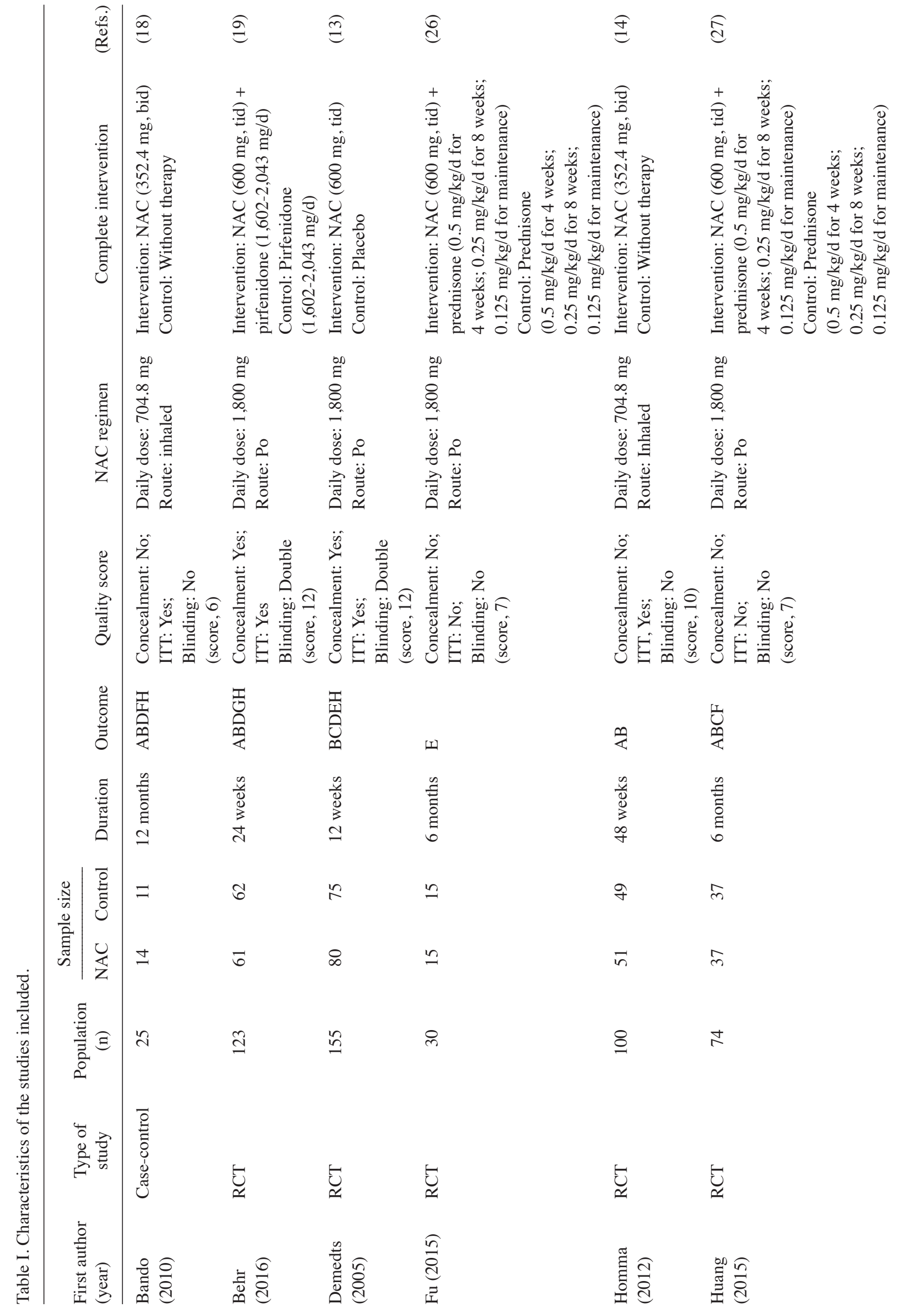




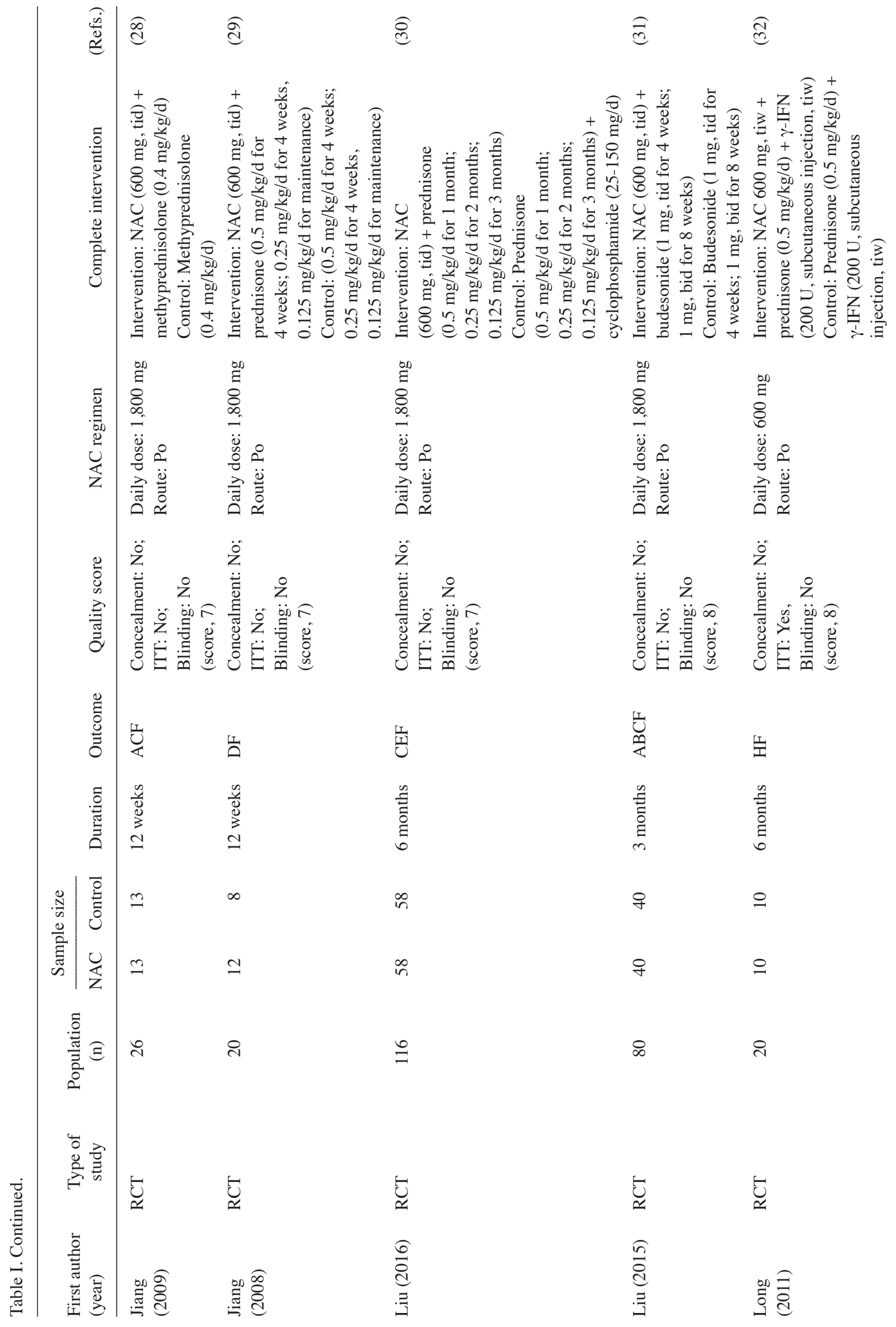




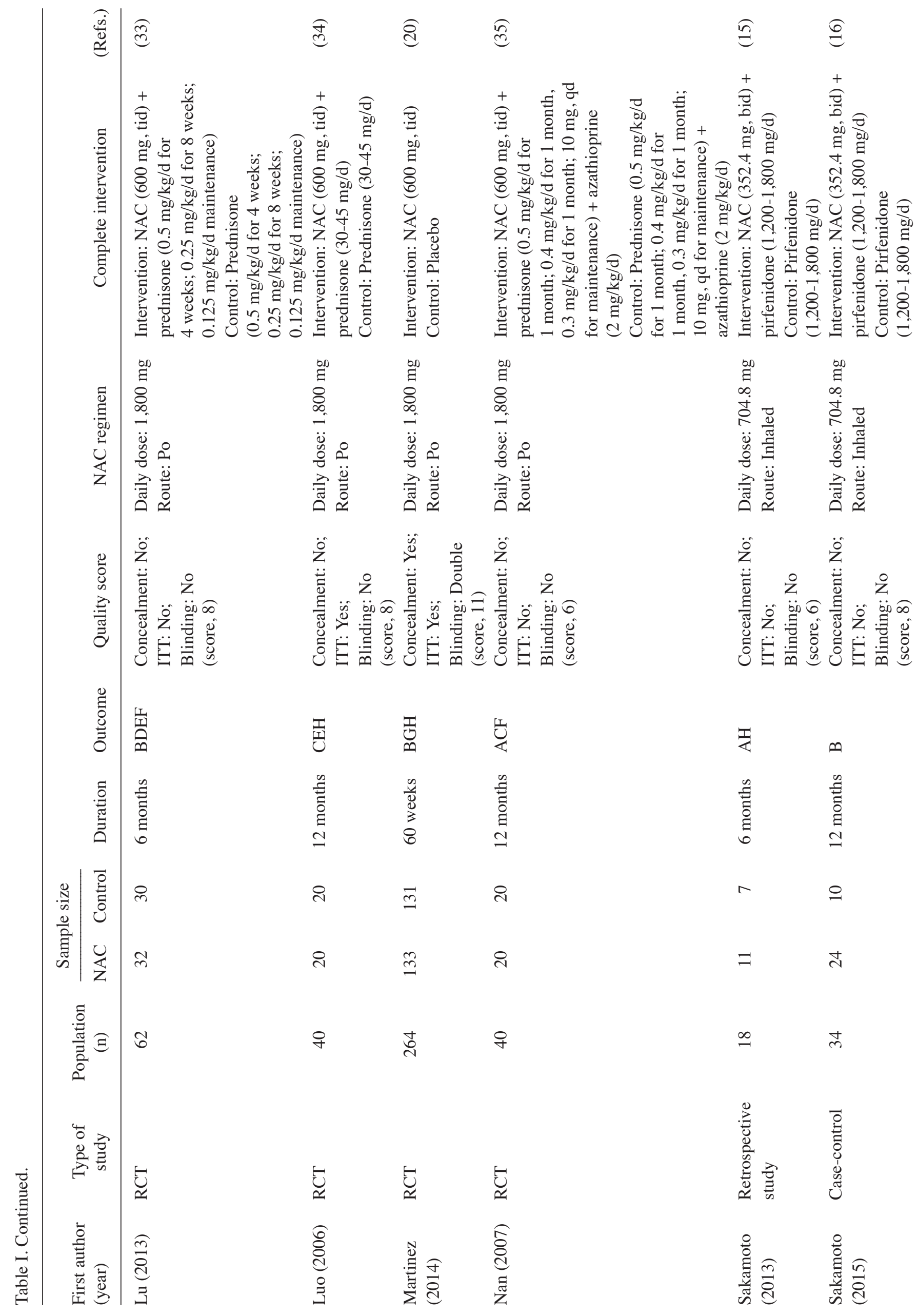




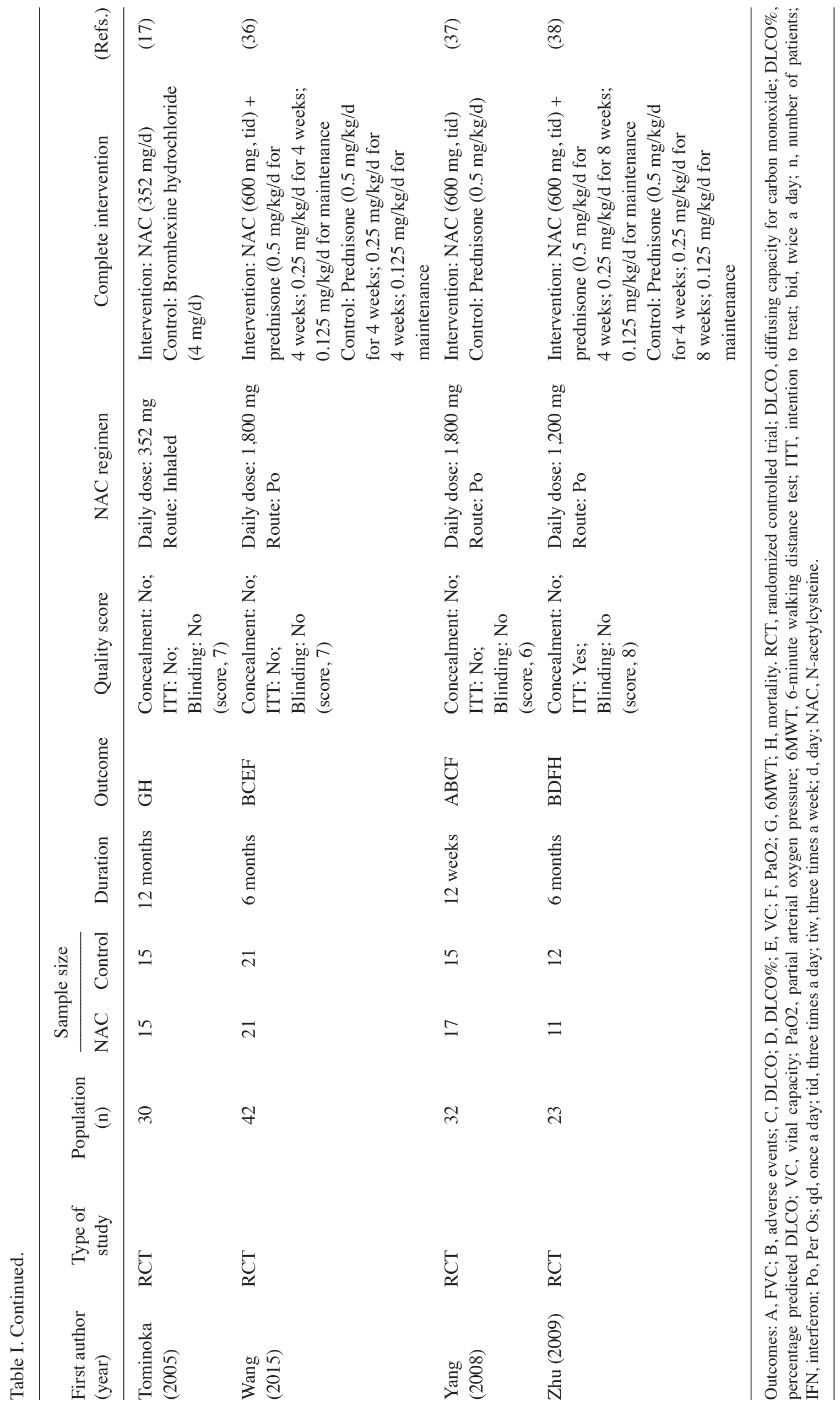



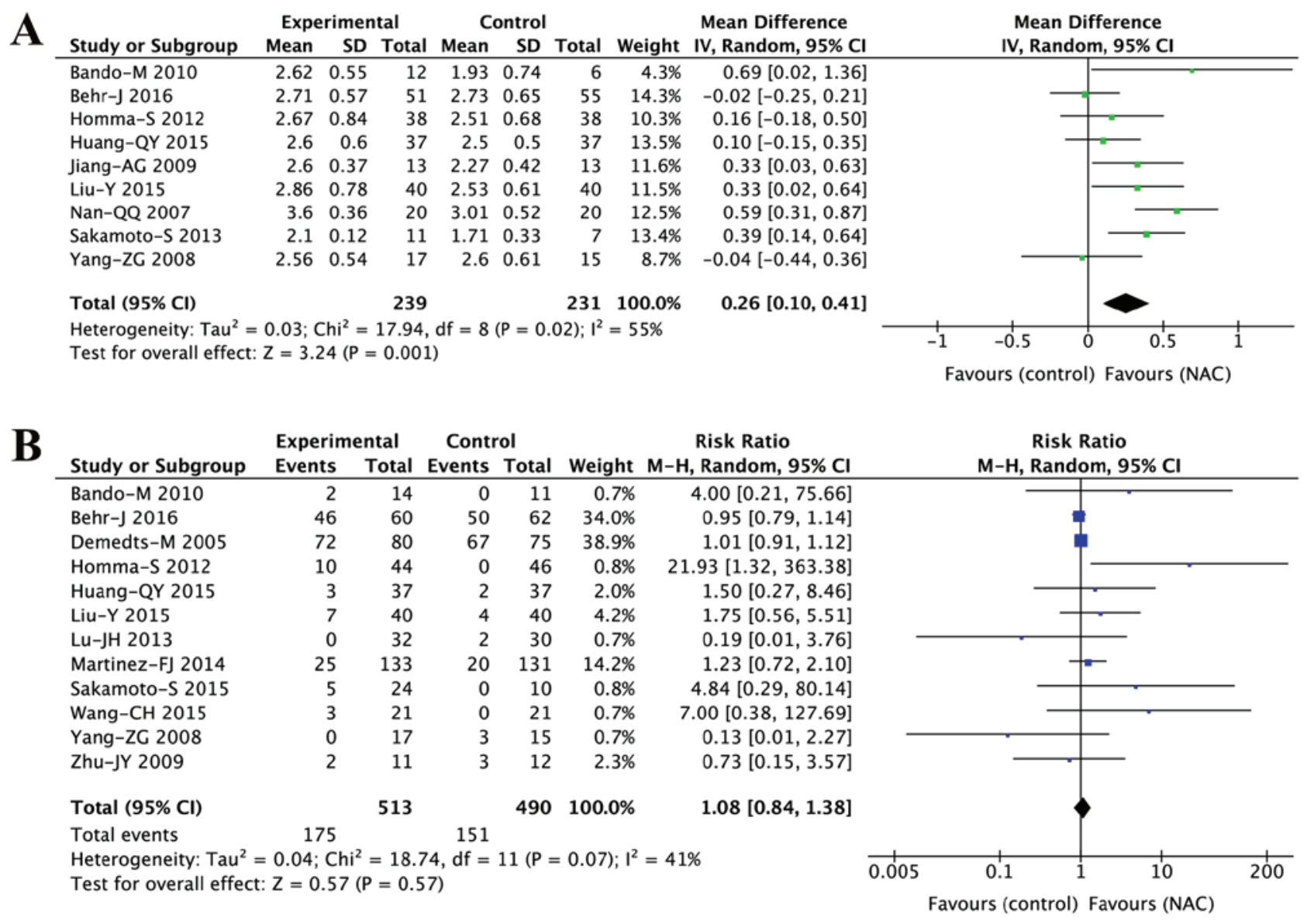

Figure 2. Forest plots for the meta-analysis on the efficacy and safety of NAC therapy for idiopathic pulmonary fibrosis with regard to the primary outcomes. (A) FVC and (B) adverse effects. FVC, forced vital capacity; IV, inverse variance; M-H, Mantel-Haenszel; NAC, $N$-acetylcysteine; df, degrees of freedom.

fixed-effects model was used due to homogeneity among the studies $\left(\mathrm{I}^{2}=1 \%, \mathrm{P}=0.42\right)$. The decline in DLCO was significantly lesser in the NAC treatment group compared with that in the control group (SMD, 0.41; 95\% CI, 0.21-0.61; P<0.0001; Fig. 3A).

Overall effect on DLCO\%. Of the 21 studies, 6 studies $(13,18,19,29,33,38)$ evaluated the DLCO\%. There was significant heterogeneity among the studies $\left(\mathrm{I}^{2}=54 \%, \mathrm{P}=0.05\right)$, and thus, a random-effects model was used. Pooled analysis revealed there was no significance (SMD, 0.31; 95\% CI, $-0.07-0.68 ; \mathrm{P}=0.11$; Fig. 3B).

Overall effect on $V C$. VC was reported for 393 patients in six studies $(13,26,30,33,34,36)$. A random-effects model was used $\left(\mathrm{I}^{2}=69 \%, \mathrm{P}=0.006\right)$ and the meta-analysis identified no influence of NAC therapy on the change in VC (MD, 0.06 ; $95 \% \mathrm{CI}$, -0.10 to 0.23 ; $\mathrm{P}=0.44$; Fig. $3 \mathrm{C}$ ).

Overall effect on $\mathrm{PaO}_{2} . \mathrm{PaO}_{2}$ was reported for 566 patients in 12 studies $(18,27-33,35-38)$. A random-effects model was used $\left(\mathrm{I}^{2}=67 \%, \mathrm{P}<0.001\right)$ and the meta-analysis revealed that $\mathrm{PaO}_{2}$ was significantly greater in the NAC therapy group than that in the control group (SMD, 0.62; 95\% CI, 0.30-0.94; P<0.001; Fig. 3D).

Overall effect on the change of 6MWT. Although the 6MWT was reported in four trials, only three trials reported data as means and standard deviations, which was required for statistical aggregation. A random-effects model was used $\left(\mathrm{I}^{2}=97 \%\right.$, $\mathrm{P}<0.001)$ and the meta-analysis indicated that improvement in the 6MWT result was significantly greater with NAC treatment than with control treatment (MD, 23.69; 95\% CI, 7.92-39.47; $\mathrm{P}=0.003$; Fig. 3E).

Overall effect on mortality. Mortality was also assessed for 697 patients in nine studies $(13,15,17-20,32,34,38)$. These studies indicated homogeneity (Fig. 3F), and therefore, a fixed-effects model was used $\left(\mathrm{I}^{2}=31 \%, \mathrm{P}=0.17\right)$. The results revealed that NAC treatment had no influence on mortality (RR, 1.02; 95\% CI, 0.66-1.59; $\mathrm{P}=0.92$ ).

\section{Subgroup analysis of FVC}

Monotherapy vs. combined therapy. Among the nine studies that evaluated the FVC, six trials $(15,19,27,28,31,35)$ and three trials $(14,18,37)$ involved combined therapy and monotherapy, respectively. While combined therapy including NAC significantly reduced the decline in FVC (MD, 0.28; 95\% CI, 0.09-0.46; $\mathrm{P}=0.003$ ), NAC monotherapy did not achieve a significant difference (MD, $0.19 ; 95 \% \mathrm{CI},-0.15$ to 0.52 ; $\mathrm{P}=0.27$; Fig. 4). However, there was no significant difference between combined and monotherapy ( $\mathrm{P}=0.63$; Fig. 5).

Low vs. high dose of NAC. A total of six $(19,27,28,31,35,37)$ and three $(14,15,18)$ studies used high $(1,800 \mathrm{mg}$ per day $)$ 
$\mathbf{A}$

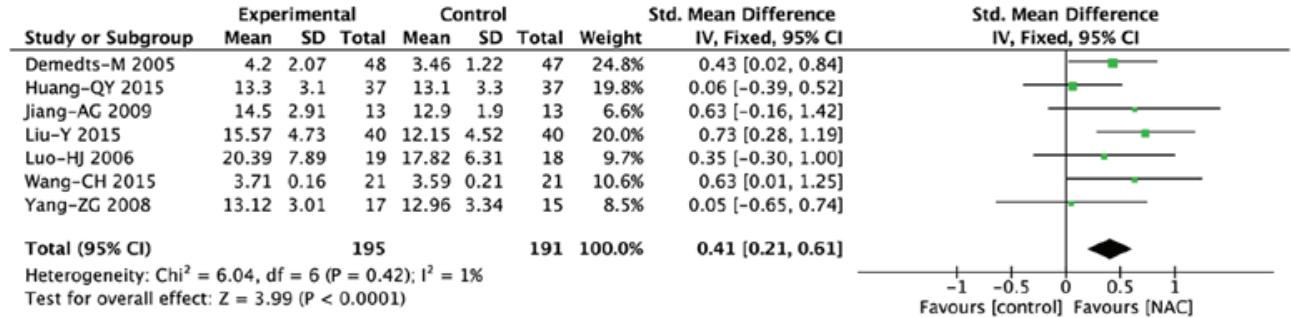

B

\begin{tabular}{|c|c|c|c|c|c|c|c|c|c|}
\hline \multirow[b]{2}{*}{ Study or Subgroup } & \multicolumn{3}{|c|}{ Experimental } & \multicolumn{3}{|c|}{ Control } & \multicolumn{2}{|c|}{ Std. Mean Difference } & \multirow{2}{*}{$\begin{array}{l}\text { Std. Mean Difference } \\
\text { IV, Random, } 95 \% \mathrm{CI}\end{array}$} \\
\hline & Mean & SD & Total & Mean & SD & Total & Weight & & \\
\hline Bando-M 2010 & 39.3 & 16.2 & 12 & 41.8 & 16.9 & 6 & $10.2 \%$ & $-0.14[-1.13,0.84]$ & \\
\hline Behr-J 2016 & 40.41 & 10.26 & 45 & 39.92 & 10.13 & 44 & $23.7 \%$ & $0.05[-0.37,0.46]$ & - \\
\hline Demedts-M 2005 & 40.85 & 14.85 & 48 & 38.75 & 14.75 & 47 & $24.2 \%$ & $0.14[-0.26,0.54]$ & \\
\hline Jiang-YQ 2008 & 73.6 & 4.7 & 12 & 64.9 & 3.2 & 8 & $8.3 \%$ & $1.99[0.86,3.12]$ & \\
\hline Lu-JH 2013 & 54.31 & 13.9 & 32 & 50.03 & 14.2 & 30 & $21.0 \%$ & $0.30[-0.20,0.80]$ & \\
\hline Zhu-JY 2009 & 70.4 & 7.2 & 11 & 67.8 & 5.8 & 12 & $12.7 \%$ & $0.39[-0.44,1.21]$ & \\
\hline \multirow{2}{*}{\multicolumn{9}{|c|}{$\begin{array}{lcrc}\text { Total }(95 \% \mathrm{Cl}) & 160 & 147100.0 \% & 0.31[-0.07,0.68] \\
\text { Heterogeneity: } \text { Tau }^{2}=0.11 ; \mathrm{Chi}^{2}=11.06, \mathrm{df}=5(\mathrm{P}=0.05) ; \mathrm{I}^{2}=55 \% & \end{array}$}} & \\
\hline & & & & & & & & 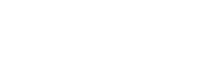 & $\begin{array}{cccc}-1 & 1 & 1 & 1 \\
-4 & -2 & 0 & 2 \\
& \text { Favours [control] Favours [N }\end{array}$ \\
\hline
\end{tabular}

C

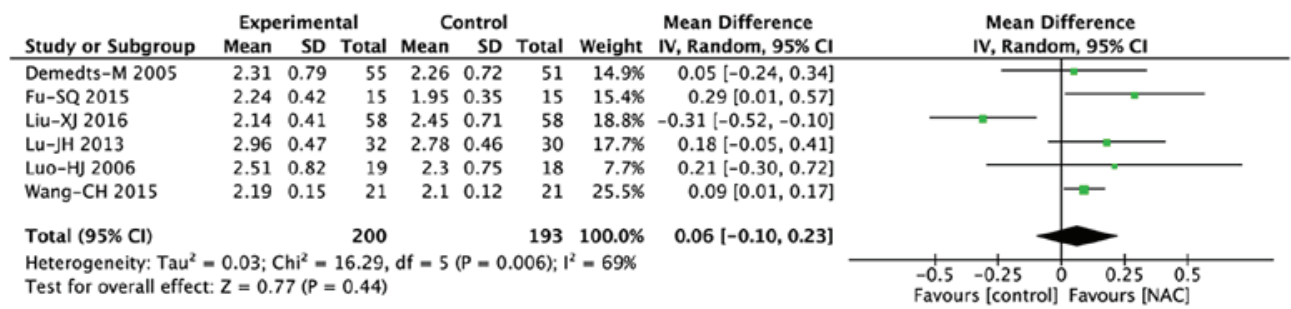

D

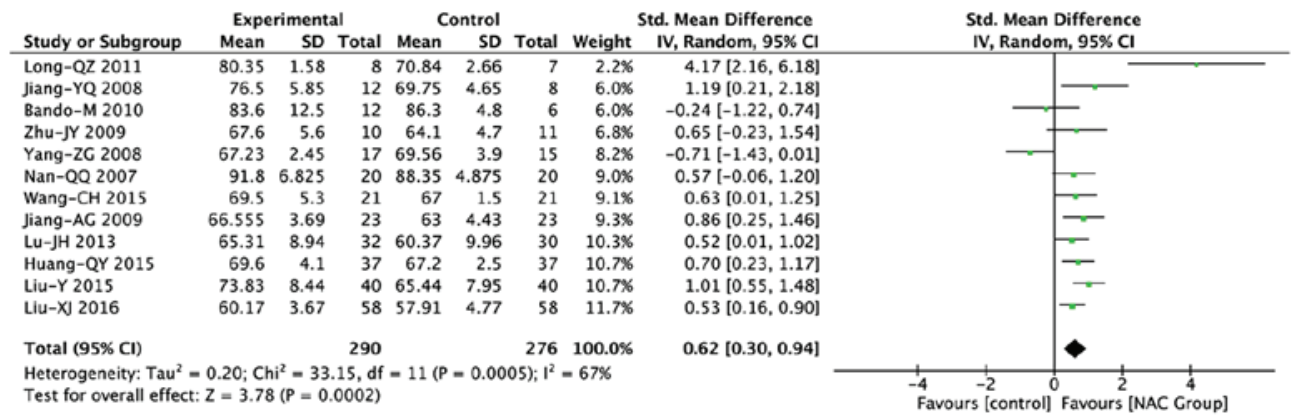

$\mathbf{E}$

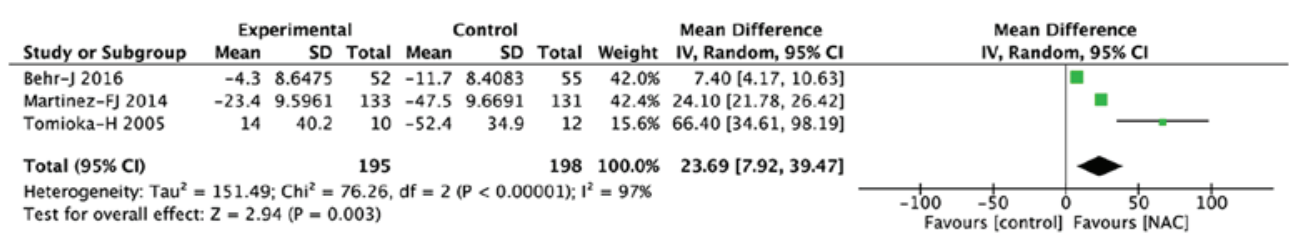

$\mathbf{F}$

Experimental Control

Risk Ratio

Risk Ratio

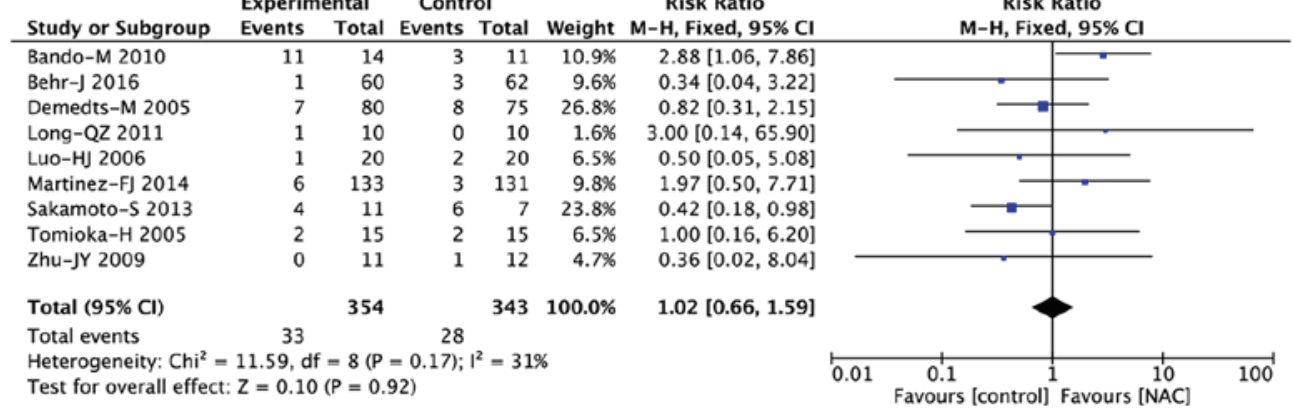

Figure 3. Forest plots for the meta-analysis on the efficacy and safety of NAC therapy for idiopathic pulmonary fibrosis (IPF) with regard to the secondary outcomes. (A) DLCO; (B) DLCO\%; (C) VC; (D) $\mathrm{PaO}_{2}$; (E) 6MWT; (F) mortality. DLCO, diffusing capacity for carbon monoxide; DLCO\%, percentage predicted DLCO; VC, vital capacity; $\mathrm{PaO}_{2}$, partial arterial oxygen pressure; 6MWT, 6-minute walking distance test; IV, inverse variance; Std., standard; M-H, Mantel-Haenszel; NAC, $N$-acetylcysteine; df, degrees of freedom. 


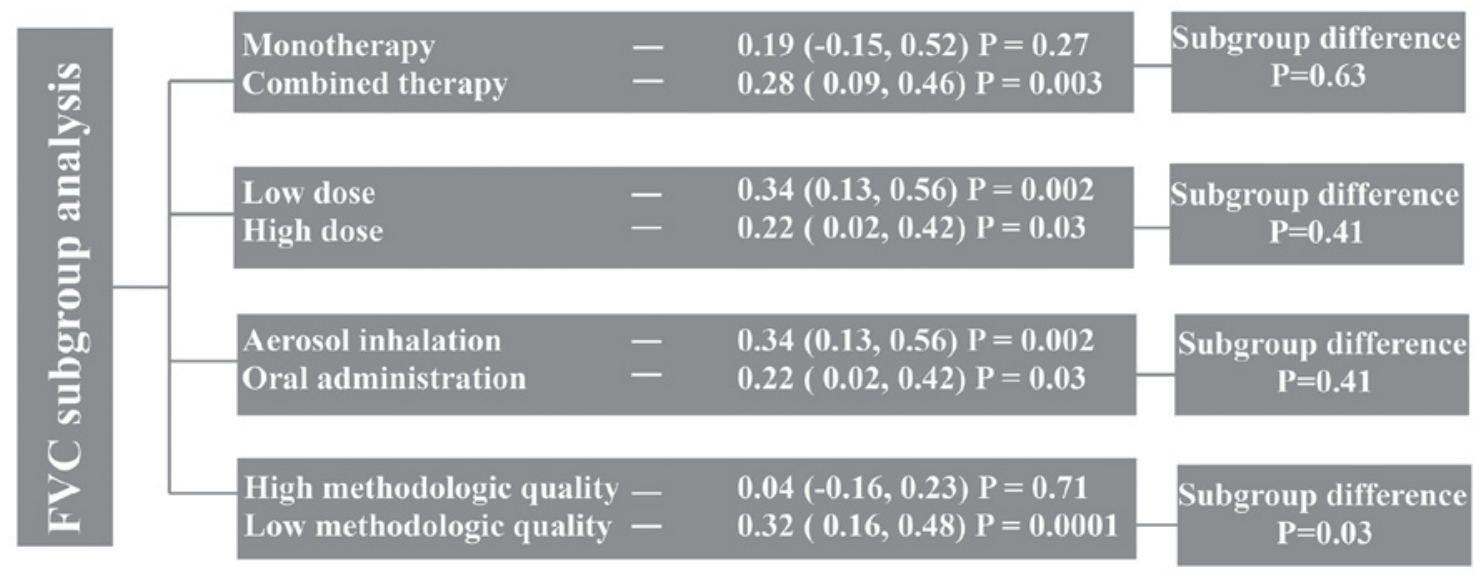

Figure 4. Summary of the subgroup analysis of the effects of $N$-acetylcysteine therapy on FVC in patients with idiopathic pulmonary fibrosis. FVC, forced vital capacity.

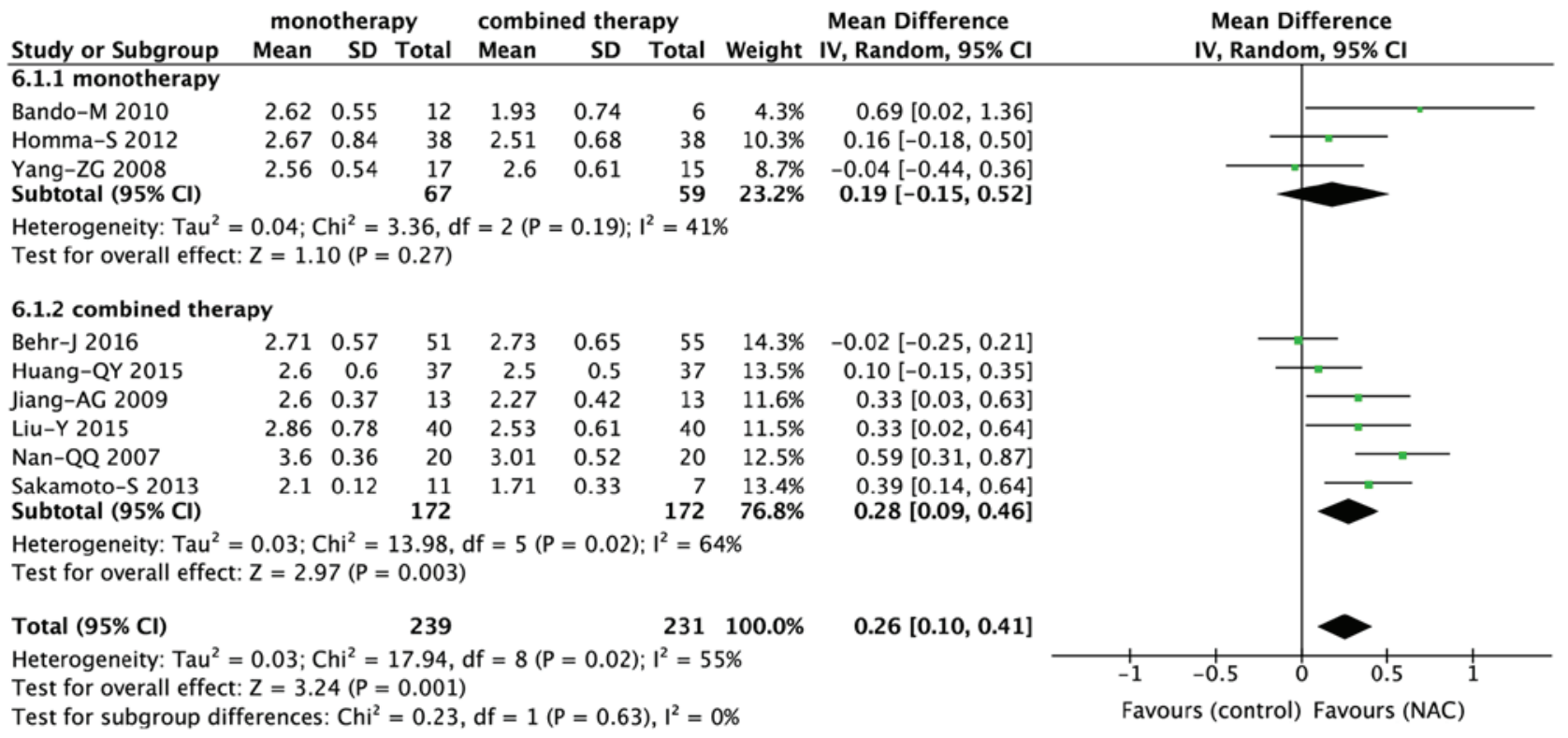

Figure 5. Subgroup analysis of the effects of NAC combined therapy and monotherapy on the forced vital capacity in patients with idiopathic pulmonary fibrosis. IV, inverse variance; NAC, $N$-acetylcysteine; df, degrees of freedom.

and low $(<1,800 \mathrm{mg}$ per day) doses of NAC, respectively. The NAC dose had no significant influence on the change in FVC (Fig. 4).

Inhaled vs. oral administration. NAC was orally administered in six studies $(19,27,28,31,35,37)$ and administered via inhalation in three studies $(14,15,18)$. The route of administration did not influence the change in FVC (Fig. 4).

Effect of the study quality on the outcomes. The mean of the methodological score was 7.9 and this was used as the cut-off score for dividing the studies into high- $(>7.9)$ and low- $(<7.9)$ quality subgroups. Among the nine studies that evaluated the FVC, two $(14,19)$ were included in the high-quality subgroup, while seven $(15,18,27,28,31,35,37)$ were included in the low-quality subgroup. The treatment effects of NAC were significantly greater $(\mathrm{P}=0.03)$ in the low-quality subgroup than in the high-quality subgroup (MD, 0.32; 95\% CI, 0.16-0.48,
$\mathrm{P}<0.001$ vs. $\mathrm{MD}, 0.04 ; 95 \% \mathrm{CI},-0.16$ to $0.23, \mathrm{P}=0.71$; Figs. 4 and 6).

Subgroup analysis of adverse effects

Monotherapy vs. combined therapy. Among the 12 studies that evaluated the adverse effects of NAC, seven $(16,19,27,31,33,36,38)$ involved combined therapy and five $(13,14,18,20,37)$ involved monotherapy. The use of NAC alone or in combination did not influence the occurrence of adverse effects (Fig. 7).

Low vs. high dose of NAC. A total of eight $(13,19,20,27$, $31,33,36,37)$ and four $(14,16,18,38)$ studies used high $(1,800 \mathrm{mg}$ per day) and low ( $<1,800 \mathrm{mg}$ per day) doses of NAC, respectively. The dose of NAC did not influence the occurrence of side effects (Fig. 7).

Inhaled vs. oral administration. A total of nine $(13,19,20,27$, $31,33,36-38)$ and three $(14,16,18)$ studies involved oral and 


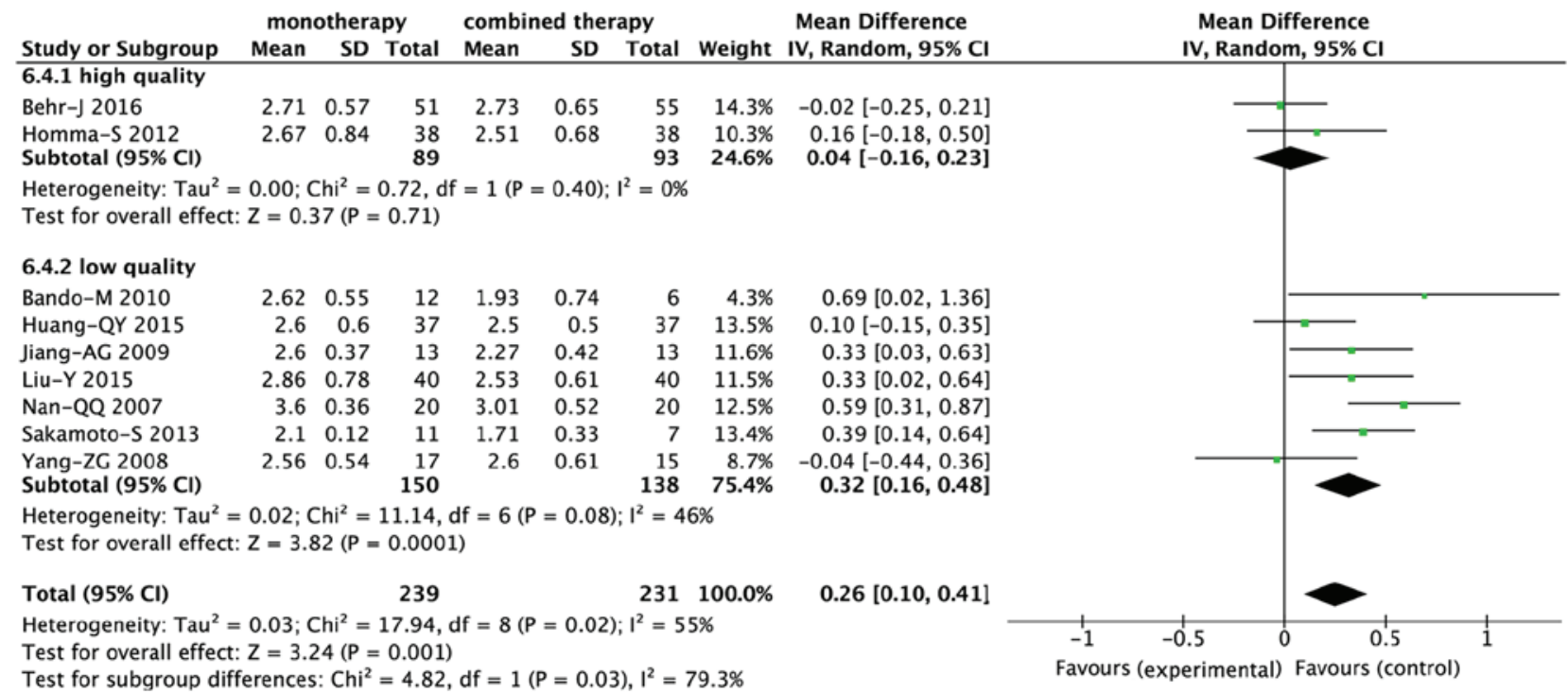

Figure 6. Subgroup analysis of the effects of NAC on the forced vital capacity in patients with idiopathic pulmonary fibrosis according to methodologic quality of the analyzed studies. IV, inverse variance; NAC, $N$-acetylcysteine; df, degrees of freedom.

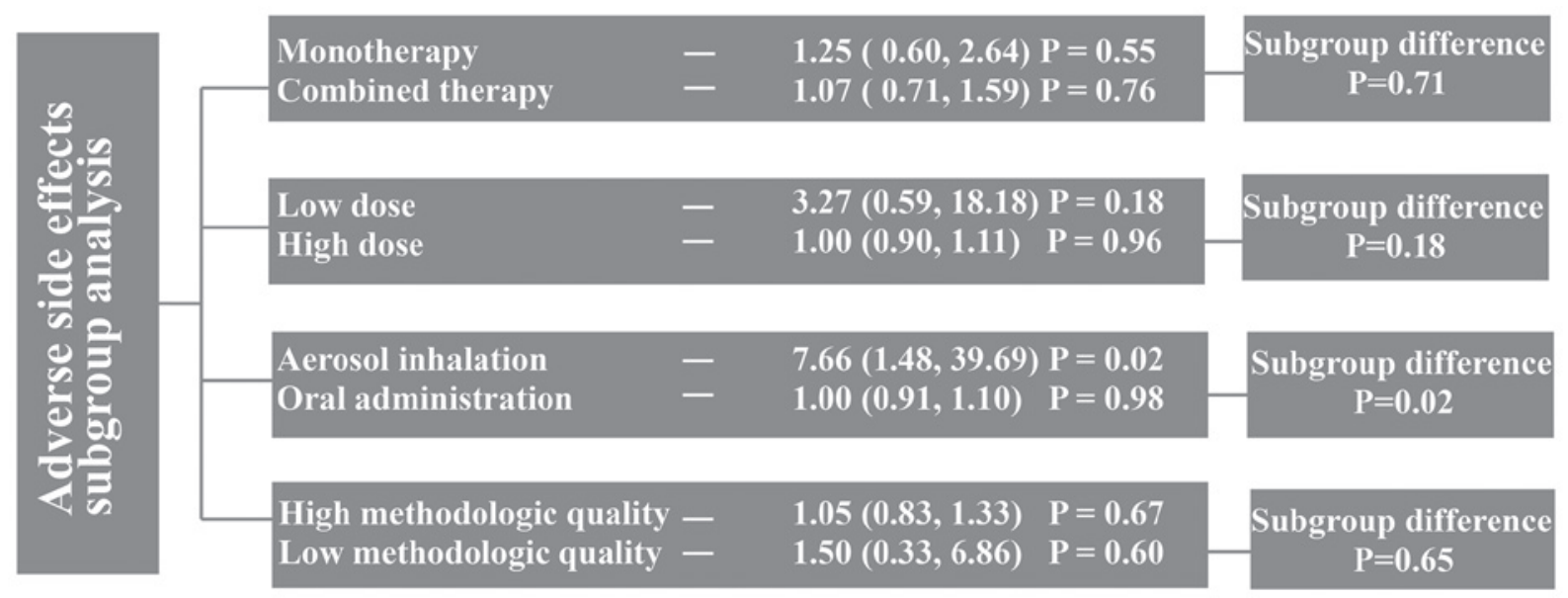

Figure 7. Summary of the subgroup analysis of adverse effects after $N$-acetylcysteine therapy for idiopathic pulmonary fibrosis.

inhaled routes of NAC administration, respectively. The incidence of adverse effects was significantly higher $(\mathrm{P}=0.02)$ in the inhalation subgroup (RR, 7.66; 95\% CI, 1.48-39.69; $\mathrm{P}=0.02)$ than that in the oral subgroup $(\mathrm{RR}, 1.00 ; 95 \% \mathrm{CI}, 0.91$ to $1.10 ; \mathrm{P}=0.98$; Figs. 7 and 8 ).

Effect of the study quality on the outcomes. The studies reporting on adverse effects were also divided into a high-qu ality $(13,14,16,19,20,31,33,38)$ and a low-quality $(18,27,36,37)$ subgroup, and no significant differences were observed between the two groups (Fig. 7).

Publication bias. Funnel plots and Egger's test were used to identify potential publication bias in the included studies. The funnel plots were asymmetrical for the studies reporting adverse effects and $\mathrm{PaO}_{2}$ data (Fig. 9); this indicated a potential risk of publication bias. However, Egger's test revealed no publication bias for adverse effects $(\mathrm{P}=0.224)$ or $\mathrm{PaO}_{2}$ data $(\mathrm{P}=0.61)$.

\section{Discussion}

IPF is a progressive and fatal disease, with symptoms including cough, chronic dyspnea, fatigue and weight loss (39). Several risk factors are associated with IPF, including environmental factors, microbial agents, gastroesophageal reflux and genetic factors (2). While affected patients may benefit from certain non-pharmacological therapies, including long-term oxygen administration or lung transplantation, there are concerns regarding available pharmacological therapies. Pirfenidone (40) and nintedanib (41), two newly approved drugs, have been reported to lower the decline in FVC in patients with IPF; however, they are associated with severe adverse effects and increased mortality rates.

The current treatment options for IPF are limited. NAC, an anti-oxidant, has been used for the treatment of IPF for several years. Although the pathogenesis of IPF remains to be fully elucidated, it has been indicated that oxidative 


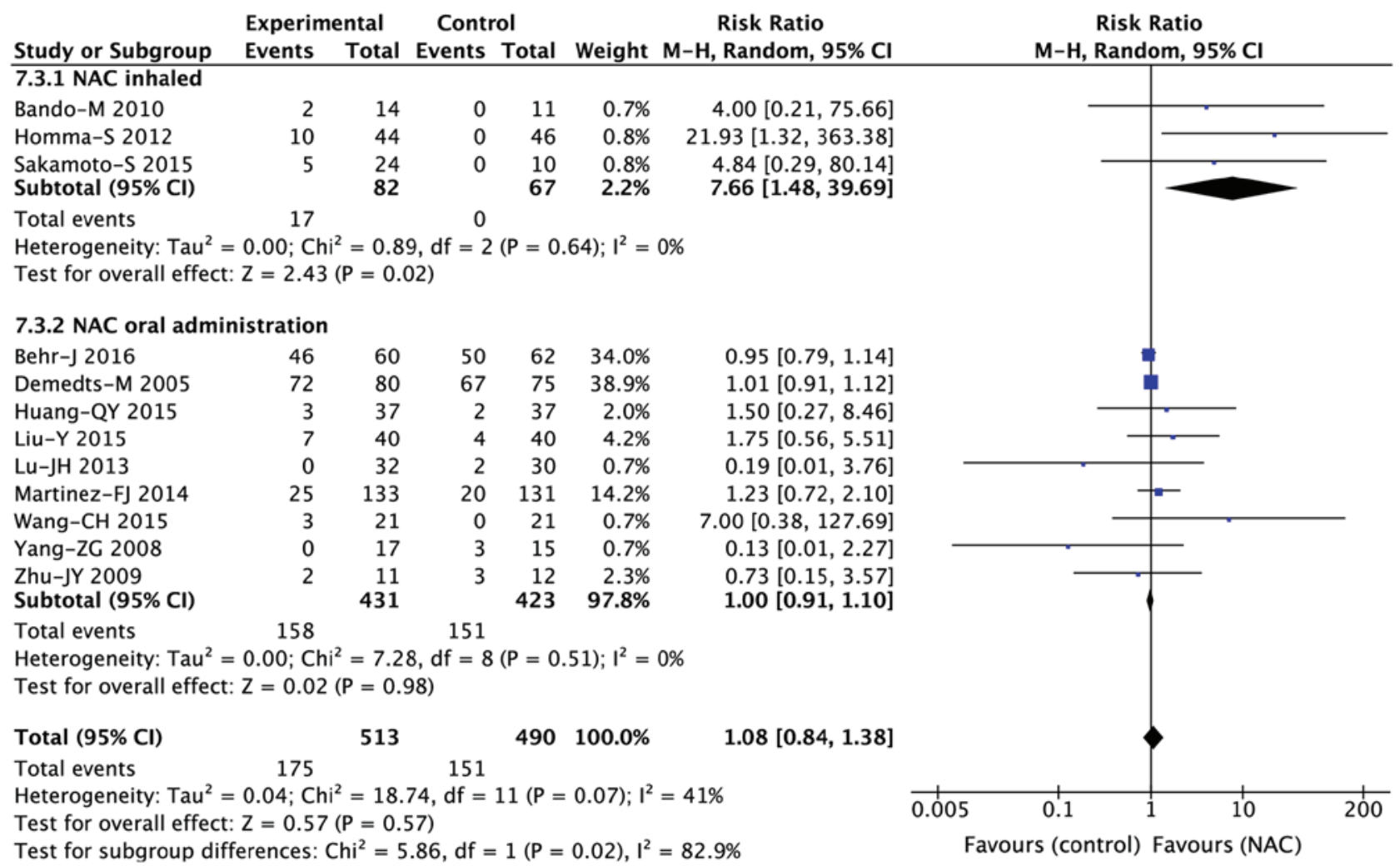

Figure 8. Subgroup analysis of adverse effects associated with NAC therapy for idiopathic pulmonary fibrosis according to the route of administration (oral vs. inhalation). M-H, Mantel-Haenszel; NAC, $N$-acetylcysteine; df, degrees of freedom.

A

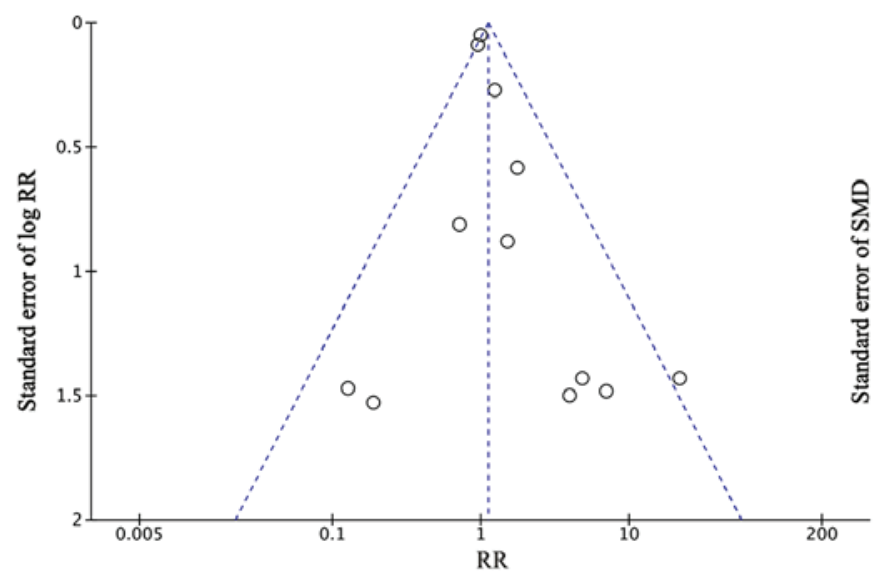

B

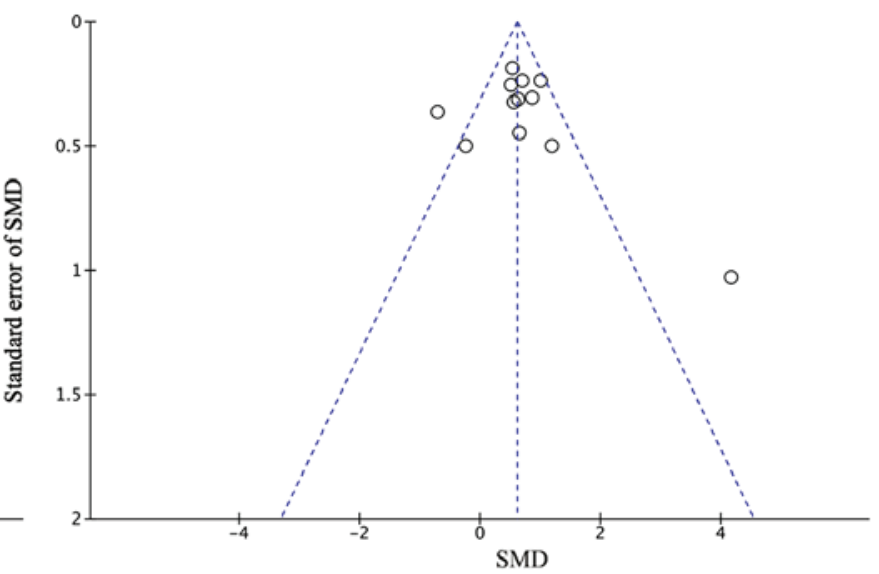

Figure 9. Funnel plots for the existence of publication bias. (A) Adverse effects and (B) partial arterial oxygen pressure. RR, risk ratio; SMD, standard mean difference.

agent-mediated alveolar epithelial cell injury, along with an abnormal fibroblast response, contributes to the development of pulmonary fibrosis $(42,43)$. NAC is a precursor of the anti-oxidant glutathione; therefore, in patients with IPF, who exhibit oxidative stress (10) and lower glutathione levels, NAC therapy may prove effective by inhibiting oxidation and restoring the redox balance. Furthermore, a study has indicated that NAC is an efficacious drug for patients with IPF associated with an rs3750920 single-nucleotide polymorphism in the gene encoding Toll-interacting protein of the TT genotype (44).
In the present updated systematic review and meta-analysis, 1,354 patients in 21 trials were evaluated regarding the efficacy and safety of NAC therapy in IPF. Two previous meta-analyses addressing the same topic have been published $(21,22)$. However, one of them only included five studies (21), while the other included 12 studies assessing anti-oxidant therapy for IPF (22). For the present meta-analysis, data for FVC, DLCO, DLCO\%, VC and 6MWT were extracted as important outcomes. Pooled analysis of the data indicated that NAC therapy significantly reduced the decline in the $\mathrm{FVC}$, a result that is different 
from those of the previous studies $(21,22)$. Furthermore, NAC significantly reduced the decline in DLCO, and improved the 6MWT results, which was in accordance with the results of Sun et al (21). Most of the studies included in the present meta-analysis also evaluated the $\mathrm{PaO}_{2}$, and the pooled analysis revealed that NAC therapy stabilized this parameter in patients with IPF. Although NAC is able to ameliorate hypoxia and protect patients with IPF from further deterioration of lung function, its adverse effects cannot be ignored. Commonly reported adverse effects in the studies analyzed included cough, dyspnea, bacterial pneumonia, diarrhea, headache, edema and abdominal pain. The incidence of cough was high in two studies $(14,18)$, in which NAC was administered via inhalation. However, the present meta-analysis identified no significant difference in the incidence of adverse effects between NAC therapy and the control treatments. Furthermore, the present analysis revealed that NAC therapy did not increase the mortality of IPF patients. However, in the PANTHER trial (45), combined therapy with NAC, prednisone and azathioprine was discontinued due to an increased incidence of mortality. When the trial was continued using a two-arm design (NAC vs. placebo), NAC therapy was indicated to have no influence on mortality (20). Of note, in most of the studies included in the present meta-analysis, NAC was administered in combination with prednisone, which may not increase mortality.

Furthermore, to address the possible heterogeneity in the primary outcomes, a hypothesis-based subgroup analysis of FVC was performed. The most important result was that NAC combined therapy significantly reduced the decline in FVC $(\mathrm{P}=0.003)$, while monotherapy did not result in a significant difference. This indicates that combined therapy may be more effective than monotherapy. However, in the PANORAMA study (19), a phase II, randomized, double-blinded, placebo-controlled study, addition of NAC to pirfenidone did not substantially alter the tolerability profile of pirfenidone, and was unlikely to have benefits for IPF. This result suggested that the combination of NAC and pirfenidone should be used with caution. The present analysis also indicated that oral administration of NAC at a high dose was beneficial in terms of FVC. However, high does should also be administered with caution. Due to limitations in the methodological quality, subgroup analysis revealed better outcomes in studies with low methodological quality than in those with high methodological quality. In the subgroup analysis on adverse effects, the only significant difference was obtained between the oral and inhalation subgroups, with the latter being associated with more adverse effects. However, the adverse effects caused by inhalation were mild to moderate and the patients were able to tolerate them. Taken together, the present meta-analysis demonstrated the efficacy and safety of NAC therapy for IPF.

Of note, the present study has certain limitations. First, none of the trials included was a randomized controlled trial, and certain studies were case-control or retrospective studies. Consequently, a certain bias associated with flaws in the methodology was inevitable. Furthermore, various important end-point variables were not evaluated, as certain outcomes were reported in a different format, e.g., delta FVC without FVC. In addition, no differentiation was made in terms of disease stage and genotypes, which may influence the efficacy of NAC therapy. With regard to adverse effects and mortality, it was not possible to determine whether all these reported adverse effects and mortality were directly associated with NAC. In the PANTHER trial, a regimen involving prednisone, azathioprine and NAC increased the rates of mortality, hospitalization and serious adverse events, while the result of the follow-up study showed that there were no significant differences between NAC and placebo in terms of mortality and acute exacerbation (45). A recent study indicated that an immunosuppressive agent affecting the telomere length in leukocytes resulted in adverse events in the patients with IPF in the PANTHER trial (46). This suggests that the safety of NAC therapy for IPF may be severely limited by the addition of azathioprine. Finally, the search performed for the present study did not include any ongoing trials and sensitivity analysis was not performed in the current meta-analysis.

In conclusion, the results of the present meta-analysis suggested that NAC therapy is a safe and effective modality that may reduce the decline in the lung function of patients with IPF. Furthermore, combined therapy including NAC and oral administration of NAC may be more effective than monotherapy and administration via inhalation. However, these results require to be interpreted with caution due to possible methodological flaws of the studies included. More well-designed, high-quality, multicenter randomized controlled trials with larger sample sizes are urgently required to clarify these results.

\section{Acknowledgements}

The authors would like to thank Dr Hailang He (Department of Respiratory Medicine, Affiliated Hospital of Nanjing University of Chinese Medicine, Nanjing, Jiangsu, China) for assistance with this updated review and Editage [www.editage.cn] for English language editing.

\section{Funding}

This study was finically supported by the National Nature Science Foundation of China (grant no. 81673936) and the Postgraduate Research \& Practice Innovation Program of Jiangsu Province (grant no. KYCX17_1311).

\section{Availability of data and materials}

All data generated or analyzed during this study are included in this published article.

\section{Authors' contributions}

XM and QW conceived and designed the study. FC and JR performed the review. FC JR and ZC analyzed the data and wrote the manuscript. XM was responsible for quality control. All authors read and approved the final manuscript.

\section{Ethics approval and consent to participate}

Not applicable. 


\section{Patient consent for publication}

Not applicable.

\section{Competing interests}

The authors declare that they have no competing interests.

\section{References}

1. Lederer DJ and Martinez FJ: Idiopathic pulmonary fibrosis. N Engl J Med 378: 1811-1823, 2018.

2. Raghu G, Collard HR, Egan JJ, Martinez FJ, Behr J, Brown KK, Colby TV, Cordier JF, Flaherty KR, Lasky JA, et al: An official ATS/ERS/JRS/ALAT statement: Idiopathic pulmonary fibrosis: Evidence-based guidelines for diagnosis and management. Am J Respir Crit Care Med 183: 788-824, 2011.

3. Richeldi L, Collard HR and Jones MG: Idiopathic pulmonary fibrosis. Lancet 389: 1941-1952, 2017.

4. Hutchinson J, Fogarty A, Hubbard R and McKeever T: Global incidence and mortality of idiopathic pulmonary fibrosis: A systematic review. Eur Respir J 46: 795-806, 2015.

5. Strand MJ, Sprunger D, Cosgrove GP, Fernandez-Perez ER Frankel SK, Huie TJ, Olson AL, Solomon J, Brown KK and Swigris JJ: Pulmonary function and survival in idiopathic vs secondary usual interstitial pneumonia. Chest 146: 775-785, 2014.

6. Wynn TA: Integrating mechanisms of pulmonary fibrosis. J Exp Med 208: 1339-1350, 2011.

7. Liu YM, Nepali K and Liou JP: Idiopathic pulmonary fibrosis: Current status, recent progress, and emerging targets. J Med Chem 60: 527-553, 2017.

8. Kliment CR and Oury TD: Oxidative stress, extracellular matrix targets, and idiopathic pulmonary fibrosis. Free Radic Bio Med 49: 707-717, 2010.

9. Kinnula VL, Fattman CL, Tan RJ and Oury TD: Oxidative stress in pulmonary fibrosis: A possible role for redox modulatory therapy. Am J Respir Crit Care Med 172: 417-422, 2005.

10. Paliogiannis P, Fois AG, Collu C, Bandinu A, Zinellu E, Carru C, Pirina P, Mangoni AA and Zinellu A: Oxidative stress-linked biomarkers in idiopathic pulmonary fibrosis: A systematic review and meta-analysis. Biomark Med 12: 1175-1184, 2018.

11. Borok Z, Buhl R, Grimes GJ, Bokser AD, Hubbard RC Holroyd KJ, Roum JH, Czerski DB, Cantin AM and Crystal RG: Effect of glutathione aerosol on oxidant-antioxidant imbalance in idiopathic pulmonary fibrosis. Lancet 338: 215-216, 1991.

12. Hagiwara SI, Ishii Y and Kitamura S: Aerosolized administration of $\mathrm{N}$-acetylcysteine attenuates lung fibrosis induced by bleomycin in mice. Am J Respir Crit Care Med 162: 225-231, 2000.

13. Demedts M, Behr J, Buhl R, Costabel U, Dekhuijzen R, Jansen HM, MacNee W, Thomeer M, Wallaert B, Laurent F, et al: High-dose acetylcysteine in idiopathic pulmonary fibrosis. $\mathrm{N}$ Engl J Med 353: 2229-2242, 2005

14. Homma S, Azuma A, Taniguchi H, Ogura T, Mochiduki Y, Sugiyama Y, Nakata K, Yoshimura K, Takeuchi M and Kudoh S; Japan NAC Clinical Study Group: Efficacy of inhaled $\mathrm{N}$-acetylcysteine monotherapy in patients with early stage idiopathic pulmonary fibrosis. Respirology 17: 467-477, 2012.

15. Sakamoto S, Itoh T, Muramatsu Y, Satoh K, Ishida F, Sugino K, Isobe $\mathrm{K}$ and Homma $\mathrm{S}$ : Efficacy of pirfenidone in patients with advanced-stage idiopathic pulmonary fibrosis. Intern Med 52: 2495-2501, 2013

16. Sakamoto S, Muramatsu Y, Satoh K, Ishida F, Kikuchi N, Sano G, Sugino K, Isobe K, Takai Y and Homma S: Effectiveness of combined therapy with pirfenidone and inhaled $\mathrm{N}$-acetylcysteine for advanced idiopathic pulmonary fibrosis: A case-control study. Respirology 20: 445-452, 2015.

17. Tomioka H, Kuwata Y, Imanaka K, Hashimoto K, Ohnishi H, Tada K, Sakamoto H and Iwasaki H: A pilot study of aerosolized $\mathrm{N}$-acetylcysteine for idiopathic pulmonary fibrosis. Respirology 10: 449-455, 2005.

18. Bando M, Hosono T, Mato N, Nakaya T, Yamasawa H, Ohno S and Sugiyama Y: Long-term efficacy of inhaled N-acetylcysteine in patients with idiopathic pulmonary fibrosis. Intern Med 49: 2289-2296, 2010.
19. Behr J, Bendstrup E, Crestani B, Günther A, Olschewski H, Sköld CM, Wells A, Wuyts W, Koschel D, Kreuter M, et al: Safety and tolerability of acetylcysteine and pirfenidone combination therapy in idiopathic pulmonary fibrosis: A randomised, double-blind, placebo-controlled, phase 2 trial. Lancet Respir Med 4: 445-453, 2016.

20. Martinez FJ, de Andrade JA, Anstrom KJ, King TE Jr and Raghu G: Randomized trial of acetylcysteine in idiopathic pulmonary fibrosis. N Engl J Med 370: 2093-2101, 2014.

21. Sun T, Liu J and Zhao de W: Efficacy of N-Acetylcysteine in idiopathic pulmonary fibrosis: A systematic review and meta-analysis. Medicine (Baltimore) 95: e3629, 2016.

22. Kandhare AD, Mukherjee A, Ghosh P and Bodhankar SL: Efficacy of antioxidant in idiopathic pulmonary fibrosis: A systematic review and meta-analysis. EXCLI J 15: 636-651, 2016.

23. Birmingham CL: Total parenteral nutrition in the critically ill patient. Lancet 353: 1116-1117, 1999.

24. Langlois PL, Manzanares W, Adhikari NKJ, Lamontagne F, Stoppe C, Hill A and Heyland DK: Vitamin C administration in the critically ill: A systematic review and meta-analysis. JPEN J Parenter Enteral Nutr 43: 335-346, 2019.

25. Manzanares W, Lemieux M, Elke G, Langlois PL, Bloos F and Heyland DK: High-dose intravenous selenium does not improve clinical outcomes in the critically ill: A systematic review and meta-analysis. Crit Care 20: 356, 2016.

26. Fu SQ, Wang J, Yan YN and Fu SB: Effects of acetylcysteine plus prednisone for idiopathic pulmonary fibrosis patients. Clin Focus 30: 38-40, 2015 (In Chinese).

27. Huang QY: Therapeutic effect of $\mathrm{N}$-acetylcysteine combined with glucocorticoid for idiopathic pulmonary fibrosis. Chin J Clin Ration Drug Use 8: 52-53, 2015 (In Chinese).

28. Jiang AG, Lu HY and Duan DJ: Therapeutic effect of $\mathrm{N}$-acetylcysteine combined with glucocorticoid for idiopathic pulmonary fibrosis. J Clin Med Pract 13: 69-70, 2009 (In Chinese).

29. Jiang YQ and Jiang $\mathrm{H}$ : Therapeutic effect of $\mathrm{N}$-acetylcysteine 20 idiopathic pulmonary fibrosis patients. Chinese Community Doctors 10: 43-44, 2008 (In Chinese).

30. Liu XJ: Glucocorticoid combined with acetylcysteine for treating idiopathic pulmonary fibrosis in 58 cases. China Pharmaceuticals 25: 99-101, 2016 (In Chinese).

31. Liu Y: Clinical effect of budesonide atomization inhalation combined with N-Acetylcysteine on idiopathic pulmonary fibrosis. Pract J Cardiac Cereb Pneumal Vasc Dis 23: 74-77, 2015 (In Chinese)

32. Long QZ, Du J, Zhang XM, Ma W and Hui K: Curative effect of combination of interferon- $\gamma, n$-acetylcysteine and low dose prednisone on idiopathic pulmonary interstitial fibrosis. J Guiyang Med Coll 36: 465-469, 2011 (In Chinese).

33. Lu JH and Gao JZ: Therapeutic effect of N-acetylcysteine combined glucocorticoid on nitric oxide in idiopathic pulmonary fibrosis patients. Chin J Lab Diagn 17: 1692-1693, 2013 (In Chinese).

34. Luo HJ: Effect of $\mathrm{N}$-acetylcysteine on pulmonary function of idiopathic pulmonary fibrosis patients. Chin J Clin Pract Med 7: 60-61, 2006 (In Chinese).

35. Nan QQ: Influence of N-acetylcysteine on serum interlukin-13 for idiopathic pulmonary fibrosis patients. Chin J Prim Med Pharm 14: 1513-1514, 2007 (In Chinese).

36. Wang $\mathrm{CH}, \mathrm{Li} \mathrm{CH}$ and Kong B: Therapeutic effect of $\mathrm{N}$-acetylcysteine on patients with idiopathic pulmonary fibrosis. Chin J Diffic and Compl Cas 14: 129-136, 2015 (In Chinese).

37. Yang ZG, Ma XT and Wang SQ: Observation on treating effect of N-acetylcystein on idiopathic pulmonary fibrosis. J Med Forum 29: 18-20, 2008 (In Chinese).

38. Zhu JY, Zeng YQ, Yuan LJ, Chen G, Wang YL and Hu K: Study on the treatment of idiopathic pulmonary fibrosis with prednisone, N-acetylcysteine combined Captopril. J Yunyang Med Coll 28: 582-584, 2009 (In Chinese).

39. Noble PW, Barkauskas CE and Jiang D: Pulmonary fibrosis: Patterns and perpetrators. J Clin Invest 122: 2756-2762, 2012.

40. Lancaster LH, de Andrade JA, Zibrak JD, Padilla ML, Albera C, Nathan SD, Wijsenbeek MS, Stauffer JL, Kirchgaessler KU and Costabel U: Pirfenidone safety and adverse event management in idiopathic pulmonary fibrosis. Eur Respir Rev 26: 170057, 2017.

41. Richeldi L, du Bois RM, Raghu G, Azuma A, Brown KK, Costabel U, Cottin V, Flaherty KR, Hansell DM, Inoue Y, et al: Efficacy and safety of nintedanib in idiopathic pulmonary fibrosis. N Engl J Med 370: 2071-2082, 2014. 
42. Duecker R, Baer P, Eickmeier O, Strecker M, Kurz J, Schaible A, Henrich D, Zielen S and Schubert R: Oxidative stress-driven pulmonary inflammation and fibrosis in a mouse model of human ataxia-telangiectasia. Redox Biol 14: 645-655, 2018.

43. Hosseinzadeh A, Javad-Moosavi SA, Reiter RJ, Yarahmadi R, Ghaznavi H and Mehrzadi S: Oxidative/nitrosative stress, autophagy and apoptosis as therapeutic targets of melatonin in idiopathic pulmonary fibrosis. Expert Opin Ther Targets 22: 1049-1061, 2018.

44. Oldham JM, Ma SF, Martinez FJ, Anstrom KJ, Raghu G, Schwartz DA, Valenzi E, Witt L, Lee C, Vij R, et al: TOLLIP, MUC5B, and the response to $\mathrm{N}$-acetylcysteine among individuals with idiopathic pulmonary fibrosis. Am J Respir Crit Care Med 192: 1475-1482, 2015.
45. Idiopathic Pulmonary Fibrosis Clinical Research Network, Raghu G, Anstrom KJ, King TE Jr, Lasky JA and Martinez FJ: Prednisone, azathioprine, and $\mathrm{N}$-acetylcysteine for pulmonary fibrosis. N Engl J Med 366: 1968-1977, 2012.

46. Newton CA, Zhang D, Oldham JM, Kozlitina J, Ma SF, Martinez FJ, Raghu G, Noth I and Garcia CK: Telomere length and use of immunosuppressive medications in idiopathic pulmonary fibrosis. Am J Respir Crit Care Med: Dec 19, 2018 (Epub ahead of print).

(i)@ $\Theta$ This work is licensed under a Creative Commons Attribution-NonCommercial-NoDerivatives 4.0 International (CC BY-NC-ND 4.0) License. 